age and acute symptomatic seizures. Increased length of hospitalization and acute symptomatic seizures were predictors of functional disability in $23 \%$ of patients with nonfatal episodes. (Claassen J, Lokin JK, Fitzsimmons BFM et al. Predictors of functional disability and mortality after status epilepticus. Neurology January (1 of 2) 2002;58:139-142).

Regarding long-term outcome, the occurrence of status with recovery had no adverse effects in a cohort of 342 children, when followed prospectively for a mean of 72 months (Shinnar S et al. Dev Med Child Neurol 1995 (suppl 72);37:116 (abstract)). It appears that mortality with status is related particularly to the cause of the status and the association of an acute illness such as encephalitis or encephalopathy, and sometimes to inadequate therapy. Although the majority of single, generalized tonic-clonic seizures are self-limiting and no more than 2 to 4 minutes duration, a seizure lasting 5 minutes or more may progress to status and should be treated aggressively. The prevention of status and the effective treatment of status are dependent on the avoidance of delay in initiating anticonvulsant therapy, and the adherence to a well-defined protocol outlined for emergency room physicians to follow. (Leppik IE, 2000; see Progress in Pediatric Neurology III, PNB Publ, 1997;102).

\title{
VIDEO-EEG IN DIAGNOSIS OF EPILEPSY IN RETARDED CHILDREN
}

Video-EEG monitoring was used to distinguish epileptic and non-epileptic events in 193 children, mean age 9.6 years, who presented with paroxysmal symptoms of uncertain etiology at Children's Hospital, Los Angeles, CA. Diagnosis was established in 130 (67\%). Seventy (36\%) were mentally retarded (MR). Epileptic seizures were identified in 67 (51\%), non-epileptic events in $54(41 \%)$, and both epileptic and non-epileptic events in $9(7 \%)$ children. Children with MR had predominantly epileptic seizures on long studies and non-epileptic events on short studies. A diagnosis was established in $82 \%$ following long studies and in $62 \%$ with short studies. The most common seizure type was complex partial, and non-epileptic symptoms were behavioral, psychogenic, and physiological events. Children with MR were more likely than children without MR to have events during the studies, but the frequencies of epileptic and non-epileptic events were similar in the two groups. Management was modified following diagnostic studies. (Thirumalai S, Abou-Khalil B, Fakhoury T, Suresh G. Video-EEG in the diagnosis of paroxysmal events in children with mental retardation and in children with normal intelligence. Dev Med Child Neurol Nov 2001;43:731-734). (Respond: Dr Shanti Thirumalai, Children's Hospital, 4650 Sunset Blvd, Mail Stop 82, Los Angeles, CA 90027).

COMMENT. Improved diagnosis and more appropriate management following video-EEG in children with paroxysmal events should prompt more general use of this test, especially in children with mental retardation.

Eight infants with early-infantile epileptic encephalopathy (Ohtahara syndrome) had seizures that correlated with the ictal burst of the suppressionburst pattern recorded by video-EEG, in a report from the Bambino Gesu' Children's Hospital, Rome, Italy (Fusco L et al. Brain Dev 2001;23:708-714).

\section{CARNITINE LEVEL CHANGES WITH THE KETOGENIC DIET}

The effects of the ketogenic diet (KD) on carnitine levels were determined in 38 consecutive patients with epilepsy treated at Rush-Presbyterian-St Luke's Medical Center, Chicago, IL. Carnitine levels were determined at $0,1,6,12$, and 24 months of diet treatment. Reduced total plasma carnitine (TC) at diet initiation was related to multiple antiepileptic drugs (AED), but only 3 had TC deficiency (range, 
11.9-21.4 mcM). Treatment with valproic acid was not responsible for the low TC. TC was unrelated to a low blood glucose or time to achieve ketosis. A decrease in TC in the first months of diet treatment was followed by stabilization and a slight increase with long term treatment. None developed clinical signs of carnitine deficiency, and only $19 \%$ of patients received supplements of carnitine. An increased acyl/free carnitine ratio is dependent on the level of ketosis during $\mathrm{KD}$ treatment, and is not a useful measure for carnitine insufficiency. (Berry-Kravis E, Booth G, Sanchez AC, Woodbury-Kolb J. Carnitine levels and the ketogenic diet. Epilepsia Nov 2001;42:1445-1451). (Reprints: Dr E Berry-Kravis, Rush-Prebyterian-St Luke's Medical Center, 1725 West Harrison St, Suite 718, Chicago, IL 60612).

COMMENT. The majority of patients on the ketogenic diet do not develop low carnitine plasma levels, and those with low levels are asymptomatic. TC should be checked at 1 to 6 months of treatment, especially for patients with low-normal TC at $\mathrm{KD}$ initiation.

\section{PAROXYSMAL TONIC UPGAZE: AGE OF ONSET AND PROGNOSIS}

Six children who developed paroxysmal tonic upgaze (PTU) at 2.6 to 7.4 years of age were examined at least once per year for a 10 year period of followup at the Departments of Paediatrics and Ophthalmology, University of Chieti, Italy. Bouts of sudden sustained upward deviation of the eyes lasted for 10 to 20 seconds each, they appeared in small clusters, and were not associated with loss of consciousness. PTU was typically exacerbated by fatigue and relieved by sleep. Three patients were male and 3 female. Two had a febrile illness preceding the onset, 2 had a personal history of febrile seizures, $1 \mathrm{a}$ father with a history of febrile seizures, and $1 \mathrm{a}$ mother with migraine. One had an epileptiform interictal EEG, with temporo-occipital spikes, mainly in the left hemisphere. MRI, neurologic, and psychological examinations, including WISC-R or Stanford-Binet Intelligence Scales were normal. Episodes of PTU resolved spontaneously after 1 to 4 years, without treatment. (Verrotti A, Trotta D, Blasetti A et al. Paroxysmal tonic upgaze of childhood: effect of age-of-onset on prognosis. Acta Paediatr Nov 2001;90:1343-1355). (Respond: Dr Albert Verrotti, Department of Pediatrics-Policlinico Colle Dell'Ara, University G D'Annunzio, Via dei Vestini 5, IT-66013 Chieti, Italy).

COMMENT. The first description of the syndrome as "benign paroxysmal tonic upgaze of childhood" was reported in 4 patients by Ouvrier RA and Billson F (I Child Neurol 1988;3:177-180). Some subsequent reports have included associated neurologic abnormalities, including ataxia (Deonna $T$ et al. 1990), developmental delay, and abnormal brain MRI. Improvement following levodopa therapy in one child suggested a possible dystonia (see Progress in Pediatric Neurology II, PNB Publ, 1994;p146). Most patients have an early spontaneous resolution of the disorder. The authors suggest that the outcome may be age-dependent, their older patients having a more benign form of the syndrome than that described in younger infants. An immature cortico-mesencephalic control of vertical gaze is postulated as the pathophysiology.

\section{MOVEMENT DISORDERS}

\section{RIGHT FRONTAL WHITE MATTER AND TOURETTE SYNDROME}

An MRI volumetric analysis of frontal and nonfrontal gray and white matter was performed in 11 boys with Tourette syndrome (TS) only, 14 with TS + ADHD, 12 with ADHD only, and 26 healthy boys, at the Kennedy Krieger Institute, 